\title{
Analysis of the Electric Arc in Low Voltage Circuit Breakers
}

\author{
A. Iturregi ${ }^{1}$, E. Torres ${ }^{2}$, I. Zamora $^{2}$ \\ ${ }^{1}$ Department of Electrical Engineering \\ EUITMOP, University of the Basque Country \\ Colina Beurko s/n, 48901 Barakaldo (Spain) \\ Phone/Fax number: +34 94601 4979/7800, e-mail: araitz.iturregi@ehu.es \\ ${ }^{2}$ Department of Electrical Engineering \\ ETSI, University of the Basque Country \\ Alameda Urquijo s/n, 48001 Bilbao (Spain) \\ Phone/Fax number: +34 94601 7332/4200, e-mail: esther.torresi@ehu.es, inmaculada.zamora@ehu.es
}

\begin{abstract}
Low Voltage Circuit Breakers (LVCBs) play an important role in electric power systems as they are responsible for switching on and off electric currents, as well as for safety of equipment and human beings. Their working base is the electric arc that appears between their contacts when establishing or interrupting the electric current in the circuit. This electric arc is a complex phenomenon where lots of physic interactions take place in a very short time. Therefore, the optimization of the operation of LVCBs makes necessary a deep understanding of the phenomena involved in the appearance of the electric arc. This knowledge can be achieved by means of modelization and simulation tools. This option allows us to obtain the evolution of physical magnitudes which would be difficult to measure in laboratory tests. The aim of this paper is to describe the phenomena of the electric arc in LVCBs, as well as the specification of the mathematical, physical and software needs for its modelization and simulation.
\end{abstract}

\section{Key words}

Low Voltage Circuit Breaker (LVCB), Electric arc, Modelization, Simulation.

\section{Introduction}

A Circuit Breaker (CB) is a switching device which can open or close a circuit in a small fraction of time, being its purpose the establishment or interruption of the circulation of current through the circuit under usual or unusual working conditions.

The interruption process of the current in a CB is always carried out by an electric arc. This process begins when the movable contact starts to separate. As a consequence, the contact area between contacts decreases and current density gets larger, until the energy associated with this process causes the metal of the contacts to begin to vaporize and an arc appears. In spite of the existence of a physical separation of the switching contacts, the established arc makes possible that current continues flowing. Thus, the current interruption involves the extinction of the electric arc, which is achieved when the interrupting medium between contacts becomes again an isolating medium [1-3].

In this paper the characteristics of the electric arc are described with the aim of characterizing the interruption process in low voltage devices. In addition, an overview of the most important models and simulation methods are exposed, as well as the last trends in the development of low voltage arc simulation. Finally some examples are shown [4-6].

\section{Electric arc phenomena in LVCB}

Physically, the arc is an incandescent gas column, with an approximate straight trajectory between electrodes (anode and cathode) and temperatures over 6000 and $10000{ }^{\circ} \mathrm{C}$. Metallic contact surfaces are also incandescent and a reduction in the cross-section of the arc is observed near them. This way, three regions can be defined: a central zone or arc column and the anode and the cathode regions (Figure 1).

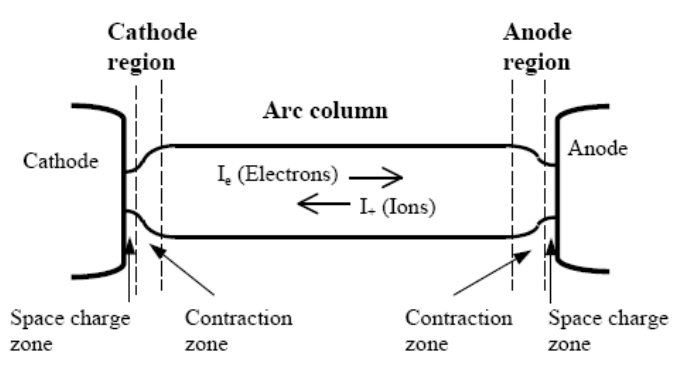

Figure 1. Electric arc structure [2]

Regarding the creation of the arc, two phenomena have to be taken into account. On one hand, conduction of electricity by gases. On the other hand, the emission of electrons by metals. Switching devices are based on the 
separation of a pair of contacts surrounded by an insulating medium, generally air for LVCB. During the opening process, ionization of the intercontact gap is produced and so it becomes conductor, appearing an electric arc that carries the electrical current. The ionization grade of the intercontact gap depends strongly on the temperature and pressure conditions, but also on the ionization potential. On this way, electric arc can be defined as a plasma column of ions and electrons from the insulating medium. However, electrons existing in the electric arc do not come only from the ionization process of the intercontact gap; they are also emitted by the metallic contacts. Free electrons in metals can be set free either by subjecting the material to a high temperature rise (thermionic emission) or to an intense electric field (field emission).

Due to those ionization processes, electric arc behaves as a conductor, being the conductivity of the plasma column in the arc between $10-100 \mathrm{~S} / \mathrm{cm}$, depending on the temperature value. To achieve its extinction, it is necessary to reduce its ionization grade as well as its conductivity, which can be made by means of intense cooling, as these properties depends strongly on the temperature values [2, 7-9].

\section{Electric arc models}

Despite the complexity associated to the arc interruption process, different methods have been developed to model and simulate its behavior. Models and simulations make possible the analysis of the time evolution of some physical magnitudes that are difficult to measure in laboratory tests. This way, the effect of those parameters in the arc interruption process and the arc phenomena itself can be evaluated.

Arc electric models can be classified in different groups [10], whose main application fields are shown in Table I. Thus, the following three groups are established: physical models (PM), "Black box" models (BB) and models based on graphics and diagrams (GD).

Table I. Application field of different switching models.

\begin{tabular}{|c|c|c|c|}
\hline \multirow{2}{*}{ Problem type } & \multicolumn{3}{|c|}{ Application } \\
\hline & Development & Test & Operation \\
\hline $\begin{array}{l}\text { Physical processes' } \\
\text { understanding }\end{array}$ & $\mathrm{PM}$ & & \\
\hline $\begin{array}{l}\text { Mechanics } \\
\text { dimensioning }\end{array}$ & PM & GD & \\
\hline $\begin{array}{l}\text { Dielectric recovery } \\
\text { description }\end{array}$ & PM, GD & GD & GD \\
\hline $\begin{array}{l}\text { Influence of the arc } \\
\text { on the current }\end{array}$ & PM, GD, BB & $\mathrm{GD}, \mathrm{BB}$ & BB \\
\hline HVDC breakers & PM, GD, BB & $\mathrm{GD}, \mathrm{BB}$ & $\mathrm{GD}, \mathrm{BB}$ \\
\hline $\begin{array}{l}\text { Small inductive } \\
\text { currents }\end{array}$ & PM, GD, BB & $\mathrm{GD}, \mathrm{BB}$ & $\mathrm{GD}, \mathrm{BB}$ \\
\hline $\begin{array}{l}\text { SLF (Short Line } \\
\text { Fault) }\end{array}$ & & & $\mathrm{GD}, \mathrm{BB}$ \\
\hline $\begin{array}{l}\text { Design and } \\
\text { verification of test } \\
\text { circuits }\end{array}$ & GD & BB & \\
\hline
\end{tabular}

\section{A. Physical models}

Physical models represent in detail the physical processes that take place in the interruption process, defining them by means of mathematical equations that govern those processes. In particular, the behavior of the electric arc is represented by a set of thermodynamic and electromagnetic laws, all of them attending to the following considerations [2]:

- Plasma is chemically reactive. Therefore, besides the mass conservation equation, velocity equations of the different reactions are needed, which for local thermodynamic equilibrium, become laws of mass action.

- Since plasma is electrically conductive, the interaction with the magnetic field must be considered in the momentum equation. This magnetic field can be applied externally or generated by the current flowing through the arc.

- The heat generated in the plasma per volume unit, due to heat dissipation by Joule effect, as well as the radiation heat emitted by the arc, must be included in the conservation of energy equation.

- There is a thermodynamic equation of state for every component present in the plasma (electrons, ions, atoms and molecular species).

Given the foregoing considerations, the equations that represent the behaviour of the arc are those included below. In particular, these equations are the NavierStokes conservation equations (representing the fluid dynamics of the interruption gas) and the Maxwell equations (modelling the electromagnetic behaviour) [2, 11-13]:

- Conservation of mass. This equation represents the sum of density change in the control volume and the difference between mass flows entering and leaving the volume (1).

$$
\frac{\partial \rho}{\partial t}+\vec{\nabla} \cdot(\rho \cdot \vec{v})=0
$$

- Conservation of momentum. The left side of equation (2) represents the sum of time rate of momentum change in the control volume and the difference of momentum of flows entering and leaving the volume. The right side is expressed as the sum of a pressure gradient, surface forces acting on the volume, and terms corresponding to magnetic and gravity forces.

$$
\begin{aligned}
& \rho \frac{\partial \vec{v}}{\partial t}+\rho(\vec{v} \cdot \vec{\nabla}) \vec{v}= \\
& =-\vec{\nabla} p+\frac{4}{3} \vec{\nabla} \mu(\vec{\nabla} \cdot \vec{v})-\vec{\nabla} \times \mu(\vec{\nabla} \times \vec{v})+\vec{j} \times \vec{B}+\rho \cdot \vec{g}
\end{aligned}
$$


- Conservation of energy. This equation represents the sum of time rate of enthalpy change in the control volume, plus the difference of enthalpy of fluids entering and leaving the volume, minus the work needed for pressure change and the work performed by the pressure gradient, to be equal to the heat loss by conduction and radiation and the electric energy dissipation in the control volume. The viscous dissipation term $\Phi$ is usually neglected (3).

$$
\begin{aligned}
& \rho \frac{\partial h}{\partial t}+\rho(\vec{v} \cdot \vec{\nabla}) h-\frac{\partial p}{\partial t}-(\vec{v} \cdot \vec{\nabla} p)= \\
& =\vec{\nabla} \cdot \frac{\lambda}{c_{p}} \vec{\nabla} h-\vec{\nabla} \cdot \vec{q}_{R}+\Phi+\vec{j} \cdot \vec{E}
\end{aligned}
$$

- Maxwell equations describe the interaction between electrical and magnetic field intensity, $\vec{E}$ and $\vec{H}$ [6] (4).

$$
\begin{aligned}
& \nabla \times \vec{E}=-\frac{\partial \vec{B}}{\partial t} \\
& \nabla \times \vec{H}=j \\
& \nabla \cdot \vec{E}=\rho_{q} \\
& \nabla \cdot \vec{B}=0
\end{aligned}
$$

Where:

$\begin{array}{cl}\mathrm{t}: & \text { Time } \\ \rho: & \text { Gas density } \\ \vec{v}: & \text { Velocity of the gas } \\ \mathrm{p}: & \text { Pressure } \\ \mu: & \text { Viscosity of the fluid } \\ \vec{E}: & \text { Electric field } \\ \vec{j}: & \text { Current density } \\ \vec{B}: & \text { Magnetic flux density } \\ \mathrm{h}: & \text { Gas enthalpy } \\ \lambda: & \text { Thermal conductivity } \\ \mathrm{c}_{\mathrm{p}}: & \text { Specific heat under constant pressure } \\ \vec{g}: & \text { Gravity acceleration }\end{array}$

Thus, electric arc physical models consider the conservation laws for mass, momentum and energy for arc macroscopic elements, but extra source terms are added. On one hand Lorentz-force density term $(\vec{j} \times \vec{B})$ is added in the momentum balance and on the other hand the ohmic heating term $(\vec{J} \cdot \vec{E})$ in the energy balance. Those extra terms couple flow dynamics with electromagnetic process, described by Maxwell's equation [11-13]. Finally, an important characteristic of physical models is that they require the knowledge of plasma properties such as mass density, specific heat, viscosity, etc. These properties are strongly dependent on temperature and pressure values and, in the presence of vaporized metal or plastic, they depend also on the characteristics of the mixture.

\section{B. Black box models}

"Black box" models define the interaction between the arc and the electrical circuit during the current interruption process. In these models the most important issue is the behavior of the arc and not how the interruption process develops.

Many of these models are based on the equations proposed by Cassie and Mayr, which represent the variation in the conductance of the arc by a differential equation obtained from physical considerations and implementation of simplifications [13]. On this way, Mayr assumed that the arc has fixed cross-sectional area losing energy only by radial thermal conduction. In contrast, Cassie assumed that the arc has a fixed temperature being cooled by forced convection [14]. Thus, "black box" models are in general represented by one differential equation relating the arc conductance with magnitudes such as voltage and arc current (5) [13].

$$
\frac{1}{G} \frac{d G}{d t}=\frac{1}{T(|i|, G)} \cdot \frac{u i}{P(i, G)}-1
$$

Where:

$$
\begin{array}{ll}
\text { G: } & \text { Arc conductance } \\
\text { u: } & \text { Arc voltage } \\
\text { i: } & \text { Arc current } \\
\text { P,T: } & \text { Parameters of the model }
\end{array}
$$

$\mathrm{P}$ and $\mathrm{T}$ parameters are calculated so as to obtain a good correlation between calculated results and those obtained by testing. The different models differ in the type of functional dependence of model parameters and how they are determined. Most of these models have no physical justification.

The fundamental purpose of "black box" models is to obtain a mathematical model that represents the circuit breaker test and can be applied in predicting the behavior of the circuit breaker under different conditions [10, 15]. These models can only be applied if the particular process that takes place is governed by the conductance. In other cases, such as in the dielectric region of breakdown processes, these models are not directly applicable.

\section{Models based on graphics and diagrams}

Finally, analytic expressions and graphics can be used, which represent a correlation between parameters of the circuit and different magnitudes associated with the interruption process and the circuit breaker performance. These expressions and graphics can be obtained from tests or from the application of both physical and "black box" models.

An example is the case where the characteristics of arc voltage are obtained experimentally. These characteristics can be used to estimate the arc-circuit interaction and can be expressed as shown in equation (6) [10]: 


$$
U_{\text {arco }}=K \cdot f\left(i, P_{S}, L\right)
$$

where:
Darco: Arc diameter nozzle
$\mathrm{K}$ : Characteristic constant of the switch and dependent on the geometry, gas type and pressure
i: Instantaneous current
Ps: Stagnation pressure in the arc area
L: Arc length.

Also, from tests and arc's physical models, expression (7) can be obtained, for its use in the design of the extinguisher gas output nozzles in circuit breakers [10].

$$
D_{\text {arco }}=K \cdot i^{a} \cdot \frac{L^{b}}{P_{S}^{c} \cdot M^{d}}
$$

where:

$$
\begin{array}{ll}
\mathrm{D}_{\text {arco }}: & \text { Arc diameter nozzle } \\
\mathrm{K}: & \text { Constant dependent on the gas type and } \\
& \text { geometry. } \\
\mathrm{i}: & \text { Instantaneous current. } \\
\mathrm{L}: & \text { Arc length. } \\
\mathrm{Ps}: & \text { Stagnation pressure in the arc area. } \\
\mathrm{M}: & \text { Mach number. }
\end{array}
$$

Another example of the application of these models is the use of graphics of the dielectric recovery, after current passage through zero, as depicted in Figure 2. These graphics can be used in the analysis of the CB behaviour on a given network, to determine if the circuit is effectively interrupted or a dielectric failure occurs [10].

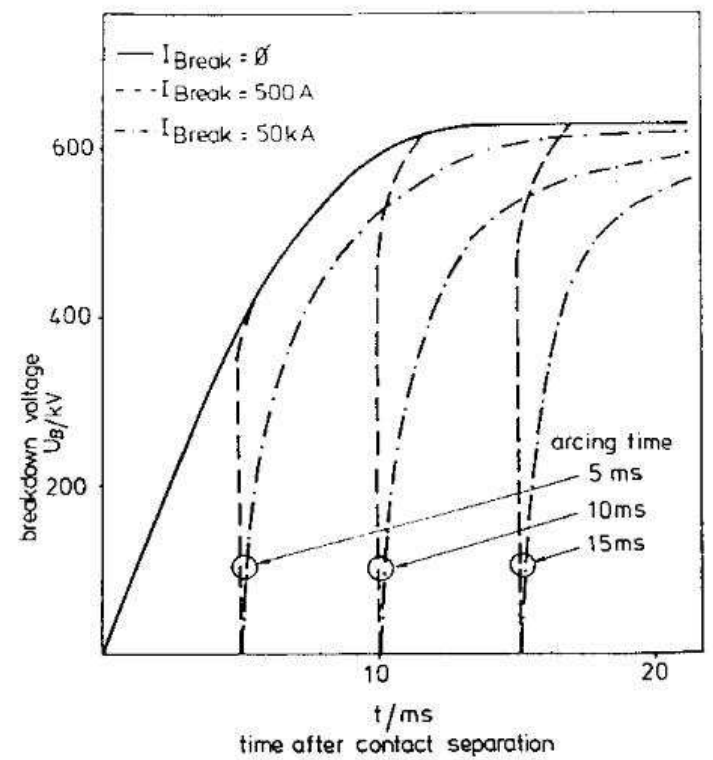

Figure 2. Dielectric properties of a CB for different current values interruption, at different arcing times [10]

The validity of this type of models is limited to the range for which its parameters have been determined experimentally. Nevertheless, they are very useful in the CB design, being also used to compare the circuit test's severity or to analyze the utility of those tests comparing them with real conditions of the network [10].

\section{Simulation}

In this section the characteristics of software tools for the simulation of the electric arc are described, as well as some examples of application.

\section{A. Software Tools}

Different software tools can be applied to simulate the behaviour of the electric arc, according to the previous classification of arc models.

Regarding "black box" models, software for simulation of power systems transients, such as ATP/EMTP and MATLAB/Simulink can be used [5]. These tools allow the analysis of power systems in the time-domain by solving the differential equations that represent the behaviour of the various components.

For physical models, software able of modelling the coupling between fluid dynamics and electric and magnetic phenomena must be used. In those models, the fluid dynamics part of the problem represented by Navier-Stokes equations is solved by means of a CFD (computational fluid dynamics) solver, whereas for electromagnetic issues Maxwell equations must be solved by a FE (finite element) solver [6]. A typical approach is the use of ANSYS, in combination with a computational fluid dynamics package, CFD, such as FLUENT or CFX. Another possibility is COMSOL Multiphysics, which allows obtaining the solution of different simultaneous physical phenomena and has recently included a specific module for plasma modeling.

Finally, as models based on graphics and diagrams are derived from the results obtained from simulations of the previous two types of models ("black box" and physical models), software tools to implement them do not really exist.

\section{B. Examples}

The aim of this section is to show some examples of different simulations, for both physical and "black box" models.

Regarding "black box" models, a significant example is the Arc Model Blockset [4]. The Arc Model Blockset is a library that currently contains seven arc models to be used in combination with the Matlab Power System Blockset. In this application, the arc is represented as a non-linear resistance, mathematically defined by a differential equation whose purpose is to the study of arccircuit interaction. In particular, the arc models incorporated in the Arc Model Blockset are: Cassie, Siemens/Habedank, KEMA, Mayr, Modified Mayr, Schavemaker and Schwarz. 
These arc models are modeled as voltage current controlled current sources, and the differential equation representing the electric arc is incorporated by means of the Simulink DEE (Differential Equation Editor). For example, Figure 3 shows the Mayr arc model included in the Arc Model Blockset, and Figure 4 shows the dialog box for the introduction of model data.

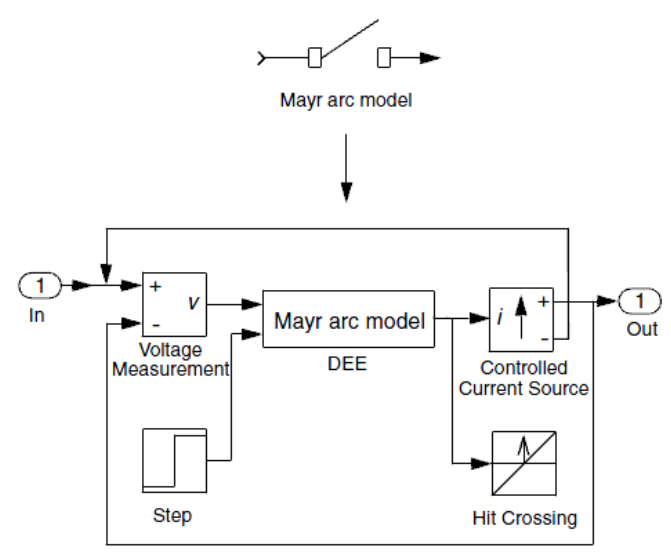

Figure 3. Mayr arc model [4]

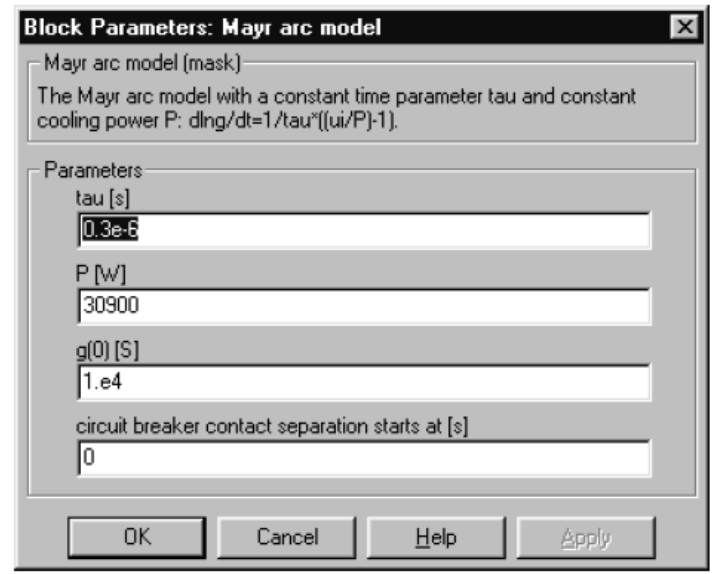

Figure 4. Arc model dialog box [4]

The proposed arc models can be connected in the circuit model as any other component. Figure 5 shows an example of the results obtained in the simulation of a circuit with the Mayr arc model.

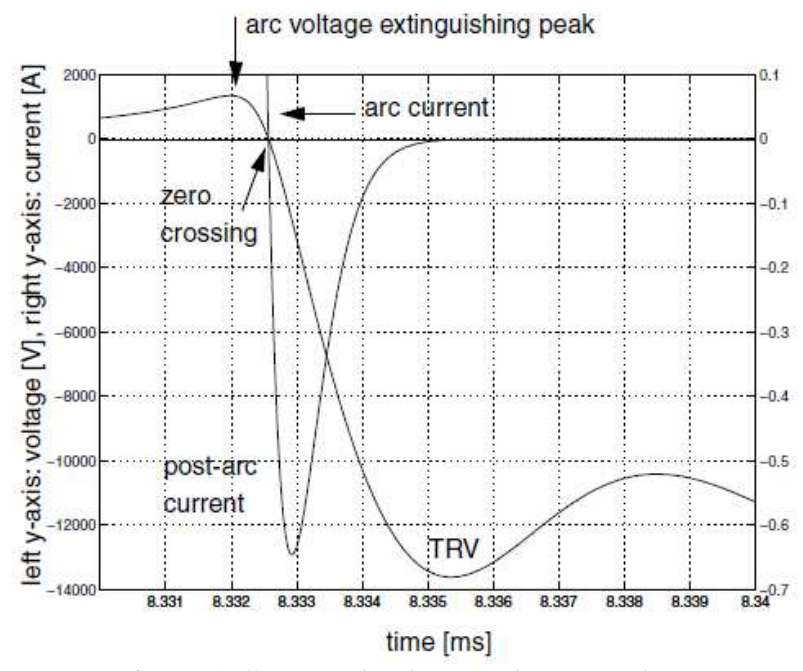

Figure 5. Computed voltage and current [4]
In Figure5, it can be observed the detailed behavior of the voltage and current around the current zero crossing when the circuit breaker contacts starts to separate at $\mathrm{t}=0 \mathrm{~s}$. As it is shown, the rapid increase in the transient recovery voltage leads to the reduction of the arc current, until it gets the zero value and the arc finally disappears.

Regarding Physical models, many researchers have proposed 2D or 3D models to study the behaviour of circuit breakers using the considerations exposed in section 3. A review of the most important trends in the development of low voltage arc simulations is included in [6]. As it is exposed, fluid dynamics and electromagnetic problems are coupled through the Lorentz force density term and the energy source term, included in the conservation equations. CFD programs offer the possibility of solving equations for the electrical and magnetic vector potential, but the presence in low voltage circuit breakers of non-linear magnetic materials makes preferable the use of a transient electromagnetic FE solver. On this way, as it was said in the previous section, a common approach for arc simulation is to use ANSYS with a CFD solver, being the simulation process developed as follows: the electrical conductivity is determined from temperature and pressure conditions provided by the CFD solver, and passed to the FE solver, which calculates the electric and magnetic fields; then, the Lorentz force density and power loss density terms are determined and sent to the CFD module, and so on.

Some key points that have to be taken into account is that detailed knowledge about plasma properties is necessary to model its behaviour with accuracy, as plasma properties depend strongly on pressure and temperature conditions and also on its composition. In addition, meshing plays a relevant role as it has a strong influence on simulation results. The increase in the number of elements leads to more accurate results but also to an increase in computation time. An example of the influence of the mesh size on simulation results is shown in Figures 6 and 7 [6].

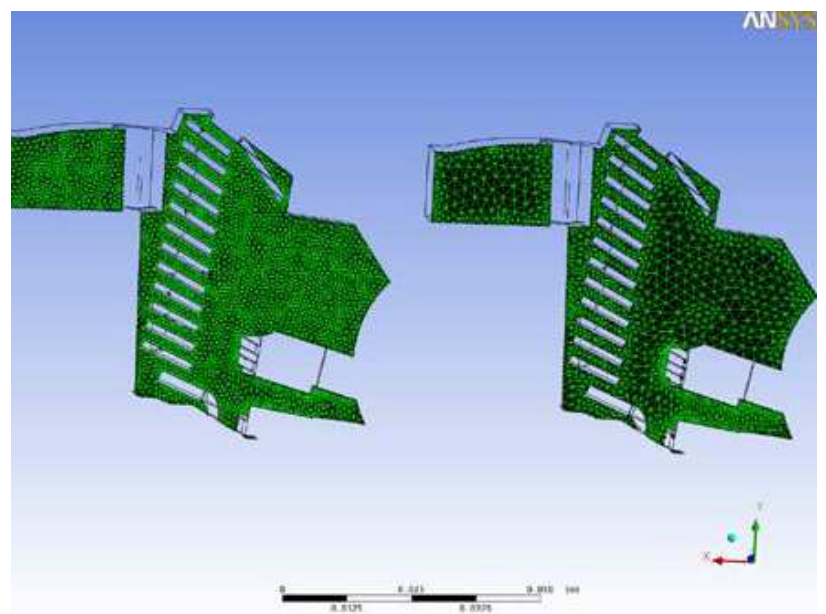

Figure 6. LVCB model in ANSYS commercial software [6]

In Figure 6, the finite element model for a LVCB is presented. There, an arc chamber with splitter plates can be seen. The model on the left has a fine meshing and the one on the right a coarse one. Figure 7 shows the 
temperature distribution obtained for both cases. As it is shown, the coarse mesh leads to a solution where temperature is rather uniform whereas a smaller element size makes possible to distinguish more temperature levels in the plasma.

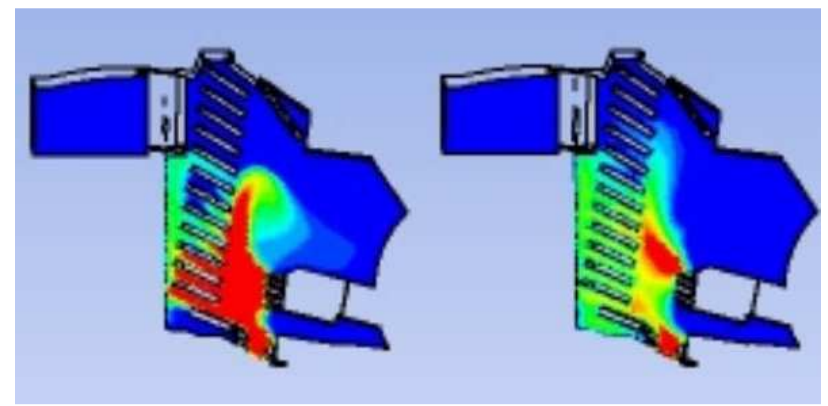

Figure 7. Temperature distribution [6]

Finally, physical arc models can be developed considering a fixed geometry, to study the time evolution of arc plasma for a given configuration. However, the motion of the movable contact can also be considered by means of the creation of moving meshes to analyse the influence of the opening velocity on the arc voltage. Figure 8 shows some results obtained when applying this approach by using FLUENT with appropriate user defined functions [16]. As it is depicted, the electric arc moves as time passes and the distance between electric contacts also increases.

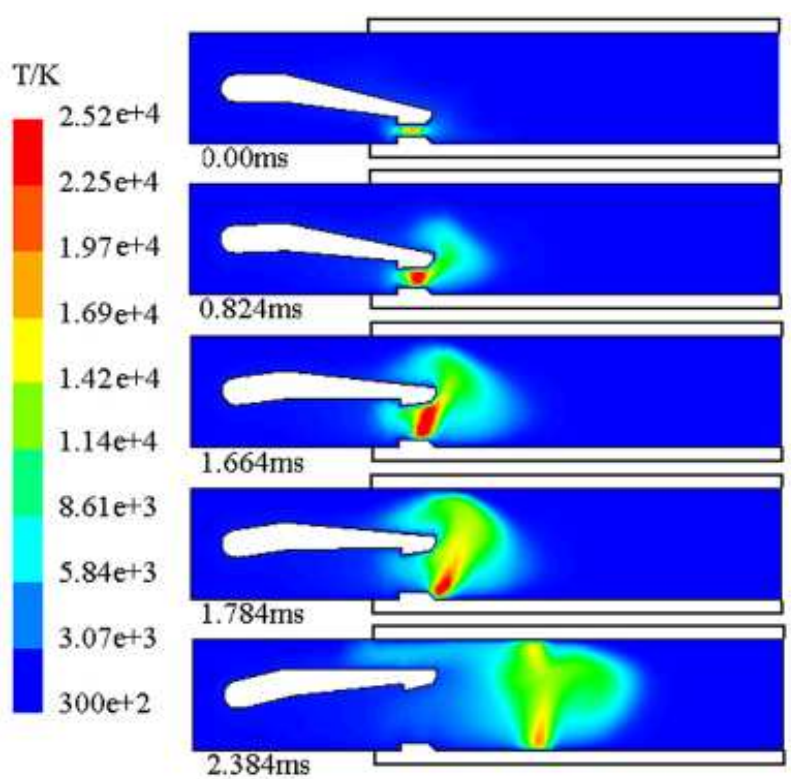

Figure 8. Temperature distribution [16]

\section{Conclusions}

The electric arc is an important phenomenon which determines the operation of LVCBs. The use of modelling and simulation tools can help to improve these devices, reducing the need of prototype development and testing and so, the cost associated to this optimization process. Three main groups of arc models can be defined: physical models, black box models and models based on graphics and diagrams. The type of model to be applied may differ depending on the purpose of the simulation.
Black-box modelling is adequate to study the arc-circuit interaction, whereas in the case of the design of new circuit breakers or to increase the understanding of the interruption process, the most appropriate, despite their complexity, are physical models.

\section{Acknowledgments}

The work presented in this paper has been supported by the Basque Government (Ref. IT532-10) and the University of the Basque Country UPV/EHU (Ref. EHU09/45).

\section{References}

[1] R. D. Garzon, High Voltage Circuit Breakers. Design and Applications. Marcel Dekker, 2002.

[2] T. E. Browne, Circuit Interruption. Theory and Techniques. Marcel Dekker, 1984.

[3] J. H. Brunke, "Circuit Breakers: Past, present and future," Electra, vol. 208, June, 2003.

[4] P. H. Schavemaker and L. Van der Sluis, "The arc model blockset," in Proceedings of the Second IASTED International Conference Power and Energy Systems (EuroPES), Crete, Greece, June 25-28, 2002, pp. 644-648.

[5] L. Van der Sluis, Transient in Power Systems. John Wiley \& Sons, 2001.

[6] M. Anheuser and C. Lüders, "Numerical arc simulations for low voltage circuit breakers," in XVIIIth Symposium on Physics of Switching Arc, Ski Hotel - Nové Mesto na Morave, Czech Republic, 2009, .

[7] P. G. Slade, Electrical Contacts: Principles and Applications. CRC Press, 1999.

[8] J. Slepian, "Extinction of an A-C. Arc," American Institute of Electrical Engineers, Transactions of the, vol. 47, pp. 1398$1407,1928$.

[9] C. H. Flurscheim, Power Circuit Breaker Theory and Design. Peter Peregrinus, 1982.

[10] W. 1. 0. SC 13, "State of art of circuit-breaker modelling," Cigre, vol. Report N¹35, 1998.

[11] F. Karetta and M. Lindmayer, "Simulation of the gasdynamic and electromagnetic processes in low voltage switching arcs," IEEE Transactions on Components,

Packaging, and Manufacturing Technology, Part A, vol. 21, pp. 96-103, 03, 1998.

[12] M. Lindmayer, "Complete simulation of moving arcs in low-voltage switchgear," in XIV Int. Conf. on Gas Discharges and their Applications, Liverpool, 2002, .

[13] M. Lindmayer, A. Mutzke, T. Rüther and M. Springstubbe, "Some aspects of arc behavior in low-voltage arc chutes," in XVIth Symp. on Physics of Switching Arc, Brno, Czech Republic, 2005, pp. 278-292.

[14] J. A. Martinez Velasco, "Representación Avanzada de Interruptores mediante el EMTP," Revista Iberoamericana Del ATP, vol. 0, pp. 1-14, November, 1998.

[15] O. M. Cassie, "Arc rupture and circuit severity," Cigre, vol. Report Nº102, 1939.

[16] Y. Wu, M. Rong, Z. Sun, X. Wang, F. Yang and X. Li, "Numerical analysis of arc plasma behaviour during contact opening process in low-voltage switching device," Journal of Physics D (Applied Physics), vol. 40, pp. 795-802, 02/07, 2007. 